\title{
EFFECTS OF MELATONIN AND PENTOXYPHILLINE TREATMENT ON NEWBORN RAT MODEL OF HYPOXIC ISCHEMIC ENCEPHALOPATHY
}

\author{
O. Soz ${ }^{1}$, M.K. Turkmen ${ }^{1}$, A. Kumral ${ }^{2}$, B. Baykara ${ }^{2}$, D.C. Yesilirmak ${ }^{2}$, H. Baskin ${ }^{2}$, O. Yilmaz ${ }^{2}$ \\ ${ }^{1}$ Adnan Menderes University, Aydin, ${ }^{2}$ Dokuz Eylul University, Izmir, Turkey
}

Perinatal asphyxia is the most important cause of neonatal mortality and serious sequel such as motor and cognitive deficits. The aim of this study is to investigate the effects of the melatonin and pentoxyphilline on neurodegeneration and cerebral nitric oxide production in a neonatal rat model of hypoxic-ischemic brain injury.

Wistar rat pups have been used in the study. Groups in the study were sham operated group (1), melatonin treated HIE-group (2), pentoxyphilline treated HIE-group (3), melatonin+pentoxyphilline treated HIE-group (4), ethanol treated HIE-group (5), vehicle-treated HIE-group (6). In hypoxia-ischemia groups, left common carotid artery was ligated on the seventh postnatal day. Two hours after the procedure, hypoxia was applied for 2.5 hour. Melatonin, pentoxyphilline and melatonin+pentoxyphilline were injected intraperitoneally after the hypoxia period. Brain nitrite levels, neuronal cell death, apoptosis were evaluated after the hypoxicischemic insult.

Melatonin and pentoxyphilline significantly diminished number of apoptotic cells in the hippocampus. Melatonin, when administered separately, significantly preserved the number of neurons in the CA2 regions of hippocampus.

Brain nitrite levels were showed that hypoxic-ischemic injury caused a significant increase in NO production. Brain nitrite levels in group 4 were not different in carotid ligated or nonligated hemispheres. Melatonin and pentoxyphilline treatment significantly decreased NO overproduction in the hypoxic-ischemic hemisphere.

These results suggest the beneficial neuroprotective effect of melatonin and pentoxyphilline combination treatment in this model of neonatal hypoxic-ischemic brain injury. 